BULLETIN Bulletin hispanique

HISPANIQUE Université Michel de Montaigne Bordeaux

109-1 | 2007

Varia

\title{
La visite du licencié Diego de Haedo dans le district inquisitorial de Saragosse (1575)
}

Vincent Parello

\section{(2) OpenEdition}

Journals

\section{Édition électronique}

URL : http://journals.openedition.org/bulletinhispanique/115

DOI : 10.4000/bulletinhispanique.115

ISSN : 1775-3821

Éditeur

Presses universitaires de Bordeaux

\section{Édition imprimée}

Date de publication : 1 juin 2007

ISBN : 978-2-85276-094-3

ISSN : 0007-4640

\section{Référence électronique}

Vincent Parello, "

La visite du licencié Diego de Haedo dans le district inquisitorial de Saragosse (1575) », Bulletin hispanique [En ligne], 109-1 | 2007, mis en ligne le 26 décembre 2013, consulté le 30 avril 2019. URL:

http://journals.openedition.org/bulletinhispanique/115; DOI : 10.4000/bulletinhispanique.115 


\title{
La visite du licencié Diego de Haedo dans le district inquisitorial de Saragosse (1575)
}

\author{
Vincent Parello \\ Université de Montpellier III
}

En 1575, lors de la visite du licencié Diego de Haedo dans le district inquisitorial de Saragosse, l'Inquisition aragonaise abandonna momentanément la lutte contre l'hérésie mahométane pour se pencher sur les délits de paroles, les délits mineurs, le luthéranisme et les délits sexuels.

En 1575, cuando la visita del licenciado Diego de Haedo en el distrito inquisitorial de Zaragoza, la Inquisición aragonesa abandonó momentáneamente la lucha contra la herejía mahometana, para castigar los delitos de palabras, las causas menores, el luteranismo y los delitos sexuales.

In 1575 when the graduate Diego de Haedo visited the inquisitorial district of Zaragoza, the Aragonese Inquisition momentarily abandoned the fight against the Mahometan heresy to dedicate itself to word, lutheranism, sexual and minor offences.

Mots-clés : Inquisition de Saragosse - Visite de district - Philippe II - ContreRéforme.

I InQuisition moderne, tribunal ecclésiastique étroitement lié à la monarchie hispanique, dispose de divers instruments, dont l'autodafé, le sambenito et la visite de district, pour assurer sa propre promotion auprès des populations nouvelles et vieilles-chrétiennes. De ces trois instruments,

$B H i$, Tome 109, n 1 - juin 2007 - p. 67 à 95. 
l'autodafé est celui qui, de toute évidence, a le plus fortement impressionné les mentalités collectives d'hier et d'aujourd'hui. Sous l'impulsion de la légende noire qui se développa sous le règne de Philippe II (1556-1598), l'autodafé devint synonyme de fanatisme religieux et de cruauté aveugle. En conférant aux sentences un maximum de publicité, l'Inquisition entendait montrer au public le triomphe du bien sur le mal, la victoire de l'orthodoxie sur l'hérésie, usant de ce que Bartolomé Bennassar a surnommé une "pédagogie de la peur ". Sur l'échafaud, dressé au milieu de la grandplace, les pénitents abjuraient leurs erreurs, les hérétiques repentis étaient réconciliés solennellement dans le giron de l'Eglise catholique, tandis que les hérétiques, restés fermes dans leur croyance, étaient conduits au bûcher pour y être brûlés par les représentants de la justice civile. Au-delà de la sentence, les carrés de lin de la honte, sur lesquels étaient inscrits les noms des accusés et la nature de leur délit et qui étaient placardés sur les murs des églises, avaient pour mission de perpétuer la mémoire de l'infamie. La présence de ces sambenitos finit par empoisonner l'atmosphère sociale des villes espagnoles et par souiller l'honneur de nombre de lignages qui, la plupart du temps, tenaient le haut du pavé. Finalement, la visite de district permettait au Saint-Office d'entrer en contact direct avec les populations et d'affirmer sa politique de la présence sur l'ensemble du territoire. Par ce biais-là, le tribunal cessait d'être une instance lointaine et abstraite pour devenir une entité palpable et concrète aux yeux des sujets de la monarchie hispanique. Même si la visite de district n'a pas frappé l'imaginaire collectif au même titre que l'autodafé et le sambenito - il s'agit, en quelque sorte, de la partie immergée de l'iceberg -, elle s'avère être une pièce essentielle de la stratégie d'occupation et de contrôle du territoire.

A la suite, entre autres, des travaux de Jean-Pierre Dedieu ${ }^{1}$ sur les visites de district, nous analyserons la visite réalisée en 1575 par le licencié Diego de Haedo «inquisidor apostólico del reyno de Aragón en todo el obispado de Jaca y parte de los de Huesca y Pamplona y del arçobispado de Çaragoça ${ }^{2}$. Nous essaierons de voir comment l'Inquisition aragonaise abandonna momentanément la poursuite du mahométisme pour se mettre au service de la Contre-Réforme en s'attaquant au luthéranisme, aux délits de paroles (blasphèmes, propositions hérétiques et propositions à caractère sexuel), aux délits mineurs (contrebande de chevaux, opposition au SaintOffice et superstition) et aux délits sexuels (sodomie, bestialité et bigamie).

1. J. P. Dedieu, «Les inquisiteurs de Tolède et la visite de district. La sédentarisation d'un tribunal (1550-1630) ", Mélanges de la Casa de Velázquez, t. XIII, 1977, pp. 235-256.

2. Archivo Histórico Nacional, Inquisición, legajo 1812, expediente 11. 


\section{CONSIDÉRATIONS GÉNÉRALES SUR LA VISITE DE DISTRICT}

A la fin du $\mathrm{XV}^{e}$ siècle, avec la sédentarisation des tribunaux locaux qui installent leur capitale au centre du district, la visite s'impose comme un nouveau mode de contrôle du territoire. Les Instructions de Torquemada de 1498, reprises par Deza deux ans plus tard, fixent l'obligation annuelle de la visite de district ${ }^{3}$. Ces visites n'ont guère évolué tout au long du XVI ${ }^{e}$ siècle. À travers les Instructions de l'inquisiteur général Valdés (1561) et la correspondance échangée entre le Conseil de l'Inquisition et les tribunaux de district, il est possible de se faire une idée assez précise de l'organisation interne de la visite de district ${ }^{4}$. Celle-ci comprend quatre temps forts : la réception de l'inquisiteur dans les localités visitées, la publication de l'édit de la foi et, le cas échéant, la proclamation de la sentence d'anathème, la collecte des témoignages et l'instruction des procès.

Accompagné d'un personnel restreint (secrétaire, notaire, alguazil), l'inquisiteur abandonne le siège de son district pour y effectuer une visite dont l'itinéraire a été défini au préalable. Dans les localités visitées, il est reçu, en grande pompe, par les autorités civiles et ecclésiastiques. Il dispose sur place d'un réseau de commissaires et de familiers pour l'aider dans sa tâche. Le samedi, un ban d'annonce est publié à la population par l'intermédiaire du secrétaire et d'un alguazil. Le dimanche, l'édit est proclamé solennellement dans l'église, en présence de l'inquisiteur et de ses ministres, de tous les notables de la ville, des autorités ecclésiastiques et de la foule anonyme. Un sermon à visée didactique et pédagogique vient parachever la lecture de l'édit. Au même moment, celui-ci est publié dans les localités avoisinantes où l'inquisiteur ne pourra se rendre en personne. La publication est confiée, dans ce cas-là, aux curés des paroisses :

Item, la dicha visita, llegado el lugar donde ha de tener asiento el dia que determinare publicar el Edicto que ha de ser domingo o fiesta de guardar, la víspera hará pregonar por orden del Secretario y Alguacil que llevare cómo dicho día se publicará el Edicto General en tal iglesia, que todos los parroquianos vayan a oille con todos los de su casa de doce años arriba, so pena de Excomunión Mayor. Publicarse el Edicto en la tal Iglesia presente el Inquisidor y los Oficiales que con él fueren y se asentará después la letura y se enviarán edictos a todos los lugares comarcanos donde el Inquisidor no oviere de ir en persona, dirigido a

3. B. Bennassar, L'Inquisition espagnole, Paris, Hachette, 1979, pp. 53-54.

4. M. Jiménez Monteserín, Introducción a la Inquisición española. Documentos básicos para el estudio del Santo Oficio, Madrid, 1980. Cet auteur retranscrit in extenso la relation d'une visite effectuée dans le district de Cuenca en 1587 (pp. 295-338). 
los Curas de los tales lugares para que los hagan leer y publicar en sus iglesias el primer día de fiesta de guardar.. ${ }^{5}$

Dans les édits, figure la liste de tous les délits que le Saint-Office entend poursuivre. À la fin du XVe et au début du XVI e siècle, le crypto-judaïsme, hérésie formelle par antonomase, y occupe une place privilégiée, mais avec le temps, d'autres formes de délits apparaissent, comme le mahométisme, le luthéranisme, l'illuminisme, la censure des livres, la superstition, les propositions hérétiques, etc. ${ }^{6} \mathrm{~A}$ la suite de la proclamation de l'édit, chaque individu est invité à venir dire s'il a "entendu dire ou vu faire quelque personne que ce soit, vivante ou morte, présente ou absente, des paroles ou des actes hérétiques, suspects, erronés, téméraires, malsonnants, scandaleux, blasphématoires " ${ }^{7}$. Au bout de quelques jours, la sentence d'anathème est proclamée solennellement dans l'église de la ville.

Au cours de la visite, l'inquisiteur doit impérativement :

1) faire rénover ou remplacer les sambenitos que le temps ou les personnes ont fait disparaittre :

Ytem, llevará el Memorial de los que obieren sido condenados $y$ reconciliados en aquel partido para, si fuere necesario renovar los sambenitos, lo pueda hacer, poniendo en los que renovare el tiempo y el delito conforme a la Instrucción, y si estuvieren renovados porná los que faltaren ${ }^{8}$.

2) traquer et punir les réconciliés, fils et petits-fils de condamnés par la ligne masculine qui auraient enfreint les pragmatiques royales et les instructions du Saint-Office en matière d'offices publics, de bénéfices ecclésiastiques et d'interdictions somptuaires :

Podrá asimismo castigar los reconciliados, hijos y nietos de condenados por linea masculina que hubieren contravenido a las Premáticas del Rey ntro Sor., Instrucciones del Santo Oficio que les prohiben traer seda, oro y armas e andar a caballo y tener oficios públicos. Y que en estos negocios no se suele dar penitencia pública ni pena de destierro, sino pena pecuniaria y si es pobre algunas penitencias espirituales ${ }^{9}$.

3) travailler en étroite collaboration avec l'Ordinaire, représentant de l'évêque dans le diocèse, en renvoyant les causes les plus importantes devant le Conseil

5. M. Jiménez Monteserín, Introducción a la Inquisición..., op. cit., p. 292.

6. Ibidem, pp. 503-563.

7. B. Bennassar, L'Inquisition espagnole, op. cit., p. 55.

8. M. Jiménez Monteserín, Introducción a la Inquisición..., op. cit., p. 291.

9. Ibidem, p. 293. 
de l'Inquisition et les informations retenues contre les commissaires et les familiers du Saint-Office devant le tribunal de district.

4) tenir un cahier avec les personnes accusées, rendre compte des amendes et des biens confisqués au receveur du tribunal et envoyer au Conseil de l'Inquisition un rapport des causes instruites avec mention du délit et de la qualité des accusés :

$Y$ vuelto el Inquisidor de la visita de la cual habrá cuaderno de penitenciados y penitenciadas, se dará cuenta al receptor de los maravedís que montaren las penitencias y se dará sumario de secuestros y se inviará relación al Consejo de las causas que se ovieren determinado desta visita y las que quedaren pendientes y la forma que se hizo la relación del acto, declarando los delitos y cualidad de los penitenciados ${ }^{10}$.

\section{AperÇU de l'aCtivité générale du tribunal de saragosse DANS LA DEUXIÈME MOITIÉ DU XVI ${ }^{\mathrm{e}}$ SIÈCLE}

Avant de passer à l'étude de la visite du licencié Diego de Haedo, il convient de dire quelques mots du tribunal de Saragosse.

Ce tribunal fut créé le 19 février 1482 sur les vestiges de l'ancienne inquisition médiévale contrôlée par les dominicains. Le 4 mai 1484 trois inquisiteurs furent nommés dans le royaume d'Aragon par fray Tomás de Torquemada, inquisiteur général des royaumes de Castille et d'Aragon depuis le 17 octobre 1483. Il s'agissait de Fray Gaspar Jutglar, provincial des dominicains, de Pedro de Epila, plus connu sous le nom de Pedro de Arbués, et d'un troisième personnage dont on ignore le nom.

Après bien des vicissitudes, le tribunal de Saragosse acquit en 1572 sa structure définitive. Il englobait les provinces actuelles de Saragosse et de Huesca, la moitié nord de la province de Teruel et l'extrême est de la province de Lérida. Parmi les principales villes : Saragosse, Huesca, Lérida, Belchite, Calanda, Jaca, Fuentes de Ebro et Saviñán. Au sud, il communiquait avec la province de Valence ; à l'est, avec la Catalogne ; à l'ouest, avec la Navarre ; et au nord, avec le Béarn ${ }^{11}$. La partie nord, qui correspond au Haut-Aragon, présente un relief très montagneux et accidenté avec des sommets atteignant les 3000 m sur la crête frontalière (Pic du Midi, Balaïtous, Vignemale, etc.). Les inquisiteurs étaient parfaitement conscients que la topographie des

10. Ibidem, p. 294.

11. Voir annexe 4. 
lieux pouvaient jouer en leur défaveur. En 1585, alarmés par la diminution drastique du nombre des familiers dans leur district, ils tenaient les propos suivants :

...que si en todas las inquisiciones es nescesario que haya bastante
número de officiales, familiares y ministros, en la de Cathaluña y
Aragón mucho más, por ser la gente aunque en lo demás buena, de
condición áspera, vengativa o vanderissa, la tierra fragosa, quebrada
y gran parte de ella maritima o frontera con Francia, a cuya causa
las denunciaciones y testificaciones se hazen con mucho miedo, y las
prissiones y demás diligencias con ordinarias reçistencias y notable
peligro ${ }^{12}$.

Aux aléas du climat et du relief venait s'ajouter un autre obstacle, non moins insurmontable : celui des mentalités!

Le tribunal de Saragosse présente une originalité par rapport au tribunal de Tolède, à partir duquel Jean-Pierre Dedieu a tenté d'élaborer un modèle généralisable à l'ensemble de la couronne de Castille ${ }^{13}$. À la suite de cet auteur, on peut distinguer quatre grandes périodes: un premier temps, caractérisé par la répression du crypto-judaïsme péninsulaire (1480-1520) ; un deuxième temps, caractérisé par la poursuite des vieux-chrétiens et des inhábiles et, dans une moindre mesure, des mahométisants et des protestants (1520-1575) ; un troisième temps, caractérisé par l'extrême variété des délits poursuivis (1575-1630) et un quatrième temps, symbolisé, entre autres, par la grande offensive menée à l'endroit des marranes portugais (1630-1720). La spécificité aragonaise, mise en évidence par Willian Monter ${ }^{14}$, est due principalement à la forte activité anti-morisque du tribunal de Saragosse, à sa juridiction spéciale en matière de délits sexuels, comme la bestialié et la sodomie, et à l'existence du délit spécifique de contrebande de chevaux avec le Béarn français. Notons aussi que le tribunal de Saragosse n’a pas été affecté par la vague de marranisme portugais au XVII siècle.

François Gomez, dans sa thèse consacrée à l'inquisition aragonaise, a exploité la série complète des relations de causes correspondant aux années 1561-1700 ${ }^{15}$. Pour la seconde moitié du XVI e siècle - période qui nous

12. Archivo Histórico Nacional, Inquisición, libro 767, folio 93.

13. J. P. Dedieu, L'administration de la foi. L'Inquisition de Tolède (XV-XVII siècle), Bibliothèque de la Casa de Velázquez, Madrid, 1992.

14. W. Monter, Frontiers of heresy. The spanish Inquisition from the Basque lands to Sicily, Cambridge, 1990.

15. F. Gómez, Le tribunal du Saint-Office de Saragosse (1561-1700). Essai d'étude quantitative, thèse de doctorat nouveau régime inédite, soutenue en 1998 à Montpellier sous 
intéresse ici -, le tribunal de Saragosse a jugé 2801 causes de foi réparties comme suit par périodes décennales : 594 entre 1561 et 1570, 484 entre 1571 et 1580, 982 entre 1581 et 1590 , et 741 entre 1591 et 1600 , soit 71 causes en moyenne par an.

\section{RÉPARTITION DES DÉLITS PAR CATÉGORIES (I 560-I600)}

1) Hérésies formelles : $(64,4 \%)$

- mahométisme (1474 causes) (52,7\%)

- luthéranisme (328 causes) (11,7\%)

- judaïsme (4) $(0,1 \%)$

2) Délits mineurs : $(14,7 \%)$

- superstition : (39) $(1,4 \%)$

- opposition au Saint-Office : (294) (10,5\%)

- contrebande : $(59)(2,1 \%)$

- divers : (9) $(0,3 \%)$

3) Délits sexuels : $(12,9 \%)$

- sodomie (175) $(6,2 \%)$

- bestialité (139) $(5 \%)$

- bigamie $(35)(1,2 \%)$

- sollicitation (15) $(0,5 \%)$

4) Délits de paroles : $(8 \%)$

- propositions : (8) $(0,2 \%)$

- blasphème : (221) $(7,8 \%)$

A la lecture de ces chiffres, il ressort que le mahométisme est le délit qui domine de loin la période avec $52,7 \%$, suivi par le luthéranisme avec $11,7 \%$ et le délit d'opposition au Saint-Office avec 10,5 \%. En revanche, le judaïsme est presque inexistant avec $0,1 \%$. Dans la seconde moitié du XVI ${ }^{e}$ siècle, les morisques eurent maille à partir avec le Saint-Office essentiellement à deux reprises : en 1581-1585 avec 409 procès et en 1596-1600 avec 247 procès. Les inquisiteurs de Saragosse craignaient que les nouveaux-chrétiens ne préparent un soulèvement général en s'alliant avec les protestants du Béarn, les morisques valenciens et les morisques grenadins déportés à la suite de la révolte des Alpujarras. Le protestantisme connut deux fortes poussées avec 143 causes au cours des années $1560-1570$ et 87 causes au cours des années

la direction de M. Raphaël Carrasco. 
1585-1590. Après la découverte de foyers protestants à Séville et à Valladolid et l'arrestation de l'archevêque de Tolède, fray Bartolomé de Carranza dans les années 1558-1559, l'Inquisition espagnole prit très au sérieux l'hérésie protestante qu'elle s'empressa d'exploiter à des fins politiques. Dans le cas aragonais, il fallait contrôler les régions limitrophes avec le Béarn français, ravagé par les guerres de religion depuis 1562. Comme le souligne François Gomez :

L'Aragon fut, sans aucun doute, la région d'Espagne où l'hérésie protestante fut la plus présente. Sa position géographique limitrophe du Béarn favorisait les contacts entre les hommes. Les relations commerciales qu'entretenaient les deux régions permettaient l'échange d'opinions et le thème religieux était souvent abordé. Contrairement à d'autres régions de la péninsule, ce ne sont pas uniquement des personnes instruites, ou des marins, des soldats et des mercenaires qui tombèrent entre les mains inquisitoriales. En Aragon, toutes les catégories socio-professionnelles sont représentées dans le délit de protestantisme ${ }^{16}$.

Finalement, le tribunal de Saragosse veilla à faire appliquer les préceptes du concile de Trente en imposant un modèle religieux et sexuel basé sur les disciplines du langage et de l'action, le refus de la Réforme et la défense du mariage chrétien. Il réprima plus particulièrement le blasphème, la sodomie et la bestialité ainsi que le délit d'opposition au Saint-Office qui regroupait des infractions variées, allant des injures et des agressions à l'égard de l'institution et de ses agents, à la "fautoría» (recel d'hérétiques, entrave au libre exercice du tribunal, etc.) en passant par les faux témoignages et les impénitents (personnes qui ne respectent pas les peines imposées par l'Inquisition).

\section{Analyse Quantitative et Qualitative De la Visite De District DU LICENCIÉ DIEGO DE HAEDO (I 575 )}

Le 17 août 1575, l'inquisiteur Diego de Haedo quitta le palais de la Aljafería à Saragosse, siège du tribunal de district, en compagnie de son secrétaire Miguel Mulga, de l'alguazil Gabriel de Herrera et du nonce Joan de Villorio et se mit en route pour la ville de Jaca où il débarqua, en grande pompe, deux jours plus tard. Un tel délai a de quoi surprendre un homme du XXI ${ }^{e}$ siècle, habitué aux moyens de transport rapides, mais il

16. Ibidem, p. 45. 
faut avoir à l'esprit qu'à cette époque-là l'espace «se mesure en journées de voyage, en ces unités de trente-cinq à quarante kilomètres qui semblent constituer la longueur normale d'une étape pédestre ${ }^{17}$. Entre le 19 août et le 30 octobre 1575, le licencié parcourut les localités de Biescas, Torla, Sallent, Canfranc, Sinués, Aragués del Puerto, Hecho, Ansó, Berdún et Luesia. Après un séjour de presque un mois à Jaca, il entreprit une seconde visite qui le conduisit respectivement à Sos, Uncastillo, Sádaba, Ejea de los Caballeros et Tauste. À chaque étape, l'édit de foi était proclamé dans la ville et les localités avoisinantes ${ }^{18}$. Au total, la visite dura quatre mois, dont un mois et demi passé dans la ville de Jaca, devenue momentanément le siège du tribunal itinérant. Il va sans dire que l'Inquisition aragonaise voulait imposer sa présence à des populations rurales et montagnardes qui vivaient à l'écart de la capitale et contrôler les frontières avec la Navarre et le Béarn français, véritable foyer réformé. La tâche était d'autant plus ardue que le Haut-Aragon entretenait des échanges permanents avec la France et que bêtes, hommes, marchandises et idées circulaient à travers les Pyrénées.

Au cours de cette visite, 105 personnes furent dénoncées par la population : 96 hommes et 9 femmes, en majorité vieux-chrétiens, dont 16 de nationalité française, originaires du Béarn ou de Gascogne. Ces personnes appartenaient de préférence au secteur tertiaire $(57,6 \%)$, mais on les trouvait également dans le secteur primaire $(24,2 \%)$ et le secteur secondaire $(18,2 \%)$. On ne peut manquer d'être surpris par le nombre élevé d'ecclésiastiques parmi elles (31,5\% du secteur tertiaire).

\section{RÉPARTITION SOCIO-PROFESSIONNELLE DES PERSONNES DÉNONCÉES}

\section{Secteur primaire (8) :}

- berger (pastor) : 2

- journalier (jornalero) : 1

- laboureur (labrador) : 5 (dont 1 mozo de labrador)

Secteur secondaire (6) :

- charpentier (carpintero) : 1

- meunier (molinero) : 1

- savetier (zapatero) : 1

- tailleur (sastre) : 3

17. J. P. Dedieu, L'administration de la foi. L'Inquisition de Tolède (XV-XVII' siècle), op. cit., p. 19.

18. Voir annexe 2. 


\section{Secteur tertiaire (19) :}

- archidiacre (arcediano de Gorga) : 1

- barbier : 1

- chanoine (canónigo de Santa Cristina en Jaca) : 1

- chapelain (capellán mayor de la seo de Jaca) : 1

- curé : 2

- évêque (obispo de Jaca) : 1

- gardien de forteresse (alcaide de Xabierrelatre) : 1

- jurat : 1

- lieutenant de gardien de forteresse : 1

- maitre d'orgue : 1

- marchand (mercader) : 2

- moine : 1

- muletier (trajinero) : 1

- seigneur : (señor de Espin ; señor de Aruex) : 2

- troubadour (juglar) : 1

- vicaire (vicario de Castejón) : 1

Parmi les chefs d'accusation, les délits de paroles (45,2\%), suivis des délits mineurs $(27,7 \%)$, des hérésies formelles $(17,3 \%)$ et des délits sexuels $(9,8 \%)$. En marge de ces dénonciations, l'inquisiteur jugea 29 procès contre des vieux-chrétiens espagnols accusés de blasphème. Ces blasphémateurs appartenaient principalement au monde rural et au clergé.

\section{RÉPARTITION SOCIO-PROFESSIONNELLE DES BLASPHÉMATEURS}

Secteur primaire : $(15)(71,5 \%)$

- berger : 1

- éleveur (ganadero) : 2

- laboureur : 12

Secteur secondaire : (1) $(0,5 \%)$

- tisserand (tejedor) $: 1$

Secteur tertiaire : $(5)(28 \%)$

- curé : 1

- gardien de forteresse (alcaide de Oliván) : 1

- piquier (soldat) gascon : 1

- recteur (rector de la parroquia de Ianuas) : 1

- vicaire : 1 
Soulignons toutefois que ces dénonciations et ces procès ne constituent qu'une infime partie de l'activité générale du tribunal de Saragosse. D'après les relations de causes, 61 procès furent instruits au cours de l'année 1575, dont six pour mahométisme, vingt pour protestantisme, dix-huit pour sodomie, deux pour bestialité, deux pour bigamie, neuf pour blasphème, un pour opposition au Saint-Office et trois pour contrebande ${ }^{19}$.

Les délits de paroles arrivent en tête des dénonciations avec 45,2\%. Les propositions, les blasphèmes et les propositions à caractère sexuel représentent respectivement $26,1 \%, 13,1 \%$ et $6 \%$.

$\mathrm{Au} X \mathrm{XVI}^{\mathrm{e}}$ siècle, les théologiens disposaient d'une gamme très étendue de termes pour rendre compte de ce genre de délits : la proposition hérétique, contraire à la vérité catholique ; la proposition erronée, qui contredit directement la foi ; la proposition teintée d'hérésie, qui affiche des thèses empruntées à d'autres confessions religieuses (protestantisme, judaïsme, etc.) ; la proposition malsonnante, qui renferme un double sens catholique et hérétique ; la proposition téméraire, qui est dépourvue de tout fondement logique et rationnel ; la proposition schismatique, qui tend à rompre l'unité de l'Eglise ; la proposition impie, qui est contraire à la piété catholique ; la proposition insultante, qui se veut diffamatoire à l'égard des représentants de l'Eglise et le blasphème, conçu comme une insulte envers Dieu ${ }^{20}$.

Le blasphème est considéré, d'un point de vue théologique, comme une faute très grave. Dans sa Guía de Pecadores, fray Luis de Granada en parle en ces termes: "Des péchés mortels, le plus grave est le blasphème, très proche des trois péchés les plus graves du Monde qui sont : l'infidélité, la désespérance et la haine de Dieu, dans l'absolu le plus grave de tous ». Saint Thomas d'Aquin y voit même une infidélité, aggravée du consentement de la volonté et de l'expression en paroles ${ }^{21}$. Les justices civiles et ecclésiastiques se montraient très rigoureuses à l'égard de ce délit. Arrachement de la langue, fouet, bannissement, prison et galères faisaient partie des peines communément appliquées aux blasphémateurs. Après une longue lutte, symbolisée en Aragon par les instructions limitatives du roi de 1500, les plaintes des Cortès de Monzón de 1510 et une nouvelle instruction limitative royale de 1514, l'Inquisition finit par retrouver sa juridiction

19. F. Gómez, Le tribunal du Saint-Office de Saragosse..., op. cit., t. II. Tableau de l'activité générale du tribunal de Saragosse par années 1561-1700.

20. M. Jiménez Monteserín, "Léxico inquisitorial ", in : Historia de la Inquisición en España y América, t. I, pp. 184-217.

21. B. Bennassar, L'Inquisition espagnole, op. cit., p. 242. 
sur les blasphèmes hérétiques -juridiction que possédait déjà l'Inquisition médiévale-et par abandonner les autres blasphèmes à la justice ecclésiastique ou civile. Quoi qu'il en soit, les inquisiteurs n'ont jamais vraiment pris ce délit au sérieux, car il ne remettait pas foncièrement en cause la foi ni le dogme catholique. Il faisait partie d'un fond folklorique et contestataire, d'un stock de formules stéréotypées que l'on utilisait dans des circonstances bien précises : état d'ébriété, colère, douleur, tristesse, jalousie, etc.

La plupart des blasphèmes tournent autour de Dieu et du Christ et, accessoirement, de la Vierge Marie et des saints. Voici quelques exemples pris au hasard. En 1571, Joan de Ayneto perdit une balle en jouant à la pelote dans la localité de Pardinilla. Dans un accès de colère, il ne put s'empêcher de prononcer un "descreo de Dios " ou un "reniego de Dios " (Annexe 3, $\mathrm{n}^{\circ}$ 9). Orosia Escartín, habitante de Varos eut ces paroles malencontreuses à la mort de sa fille :

estando llorando por la muerte de una hija suya dixo que más agravio le
avia hecho Dios a ella que a otra ninguna, que le avia llevado a su hija,
y que si tubiera alli a Dios en aquel instante, le obiera dado de porradas,
que tenia grande yra y enojo y el alma perdida (Annexe $3, n^{\circ} 13$ ).

Un dimanche matin, à la sortie de la messe, dans la localité de Luz en France, Ramón Joan invita un ami à boire un verre. Celui-ci déclina son invitation et émit le vœu de retourner à l'église pour y prier Dieu et la Vierge Marie. Ramón se mit alors dans une colère monstre et, dans un castillan mêlé de gascon, il laissa échapper un magnifique « cap de diu, ahora salís de la yglesia y ya os queréis volver a la yglesia " (Annexe 3, n 23). L'Inquisition, consciente que l'on avait affaire à des écarts de langage plutôt qu'à des propos hérétiques, se montrait très clémente envers ce genre de délits. C'est ce que prouvent les sentences prononcées en 1575 contre les vingt-neuf personnes accusées de blasphème par le licencié Diego de Haedo :

- Abjuration de levi+peine d'exil+pénitences spirituelles+amende : 1

- Abjuration de levi+peine d'exil+pénitences spirituelles : 1

- Abjuration de levi+pénitences spirituelles+amendes : 2

- Abjuration de levi+pénitences spirituelles : 1

- Pénitences spirituelles+amende : 10

- Pénitences spirituelles : 4

- Absolutions : 10

À la différence du blasphème, jugé plutôt inoffensif par les inquisiteurs, la proposition hérétique était plus sévèrement châtiée, car elle remettait en 
cause le rôle de l'institution ecclésiastique et s'attaquait aux fondements mêmes du dogme catholique. Par ailleurs, dans le contexte de l'époque, ces propositions pouvaient être teintées d'hérésie luthérienne. Dans les témoignages recueillis par l'inquisiteur, on trouve des actes sacrilèges contre des objets du culte ainsi que des critiques portant sur la confession auriculaire, la célébration de la messe, l'immortalité de l'âme, les jeûnes et les interdictions alimentaires, le culte des saints et des images, les bulles et les indulgences, les âmes du purgatoire, la virginité de Marie, l'enfer et le purgatoire, etc. Voyons quelques exemples significatifs.

Lors d'un prêche, l'évêque de Jaca, don Pedro del Frago, expliquait, par le biais d'une révélation de saint Bernard, que la confession vocale n'était pas nécessaire pour le salut de l'homme :

\footnotetext{
...hablando de los pocos que se salvaban, le oyó dezir (el testigo) que a Sant Bernardo o otro santo le avia venido una rebelaçión de la ánima de un difunto que en esse día avían muerto treynta mil personas y que no se avía salvado sino el que lo reveló y otros dos, y dixo el dicho obispo, estos que se salvaron se abian arrepentido de sus peccados y sin la confesión vocal se abian salvado, de lo cual se escandalizó mucho el testigo (Annexe 3, $\mathrm{n}^{\circ} 3$ ).
}

María Laguna, épouse de Martín Beltrán, originaire de Azín, mit volontairement en pièces une croix en cuivre avec son crucifix qu'on lui avait confiée :

le dieron una cruz de cobre con su crucifixo para que se aconsolasse y acordasse de la passión de nuestro señor, y al cabo de tres días hallaron la cruz hecha dos pedaços en el suelo, y preguntándole quién la avia rompido, dixo que ella poniéndola de punta en el suelo y echándose encima (Annexe 3, $\mathrm{n}^{\circ} 8$ ).

Andrés, habitant de Jaca, trouvait absurde de prier et de se prosterner devant un rétable qui n'était, en réalité, qu'une vulgaire construction en bois : "le oyó dezir (un testigo) que para qué hazían oración delante los retablos, que no avía allí sino tablas y madera (...) qué fruto tengo ahora de sacar dessas tablas dese retablo?" (Annexe 3, n 49). Arnao, berger gascon, prenait les bulles et les indulgences pour une véritable supercherie. C'est ce qu'il exprimait à travers une jolie paronomase : "dixo que en estas tierras dábamos dos reales por un pedaço de papel y que no eran bullas sino burlas" (Annexe 3, n 68). Comme beaucoup d'hommes de son temps, 
Joan de Camias, paysan de Santa Cilia avait du mal à croire en la virginité de Marie :

leyendo el uno en la doctrina cristiana que nuestra señora avía sido virgen antes del parto, en el parto y después del parto, respondió el dicho Joan que no creya que pariesse y quedasse virgen (...) no se lo harian creer que ninguna muger después de parida quedasse virgen (Annexe $\left.3, \mathrm{n}^{\circ} 48\right)$.

En marge du blasphème et des propositions hérétiques, il existait d'autres propositions à caractère sexuel que l'Inquisition était chargée de réprimer. Ces propositions tournaient principalement autour de deux points: la croyance que la simple fornication définie comme " une copule charnelle hors mariage entre deux personnes libres de tout lien, de consentement mutuel ${ }^{22}$ n'est pas un péché et l'idée que la fréquentation des prostituées n'est pas un péché mortel mais véniel. Il faut attendre les années 1570 pour que l'Inquisition s'attaque à ce genre de délits. Le Conseil de l'Inquisition, par le biais des Cartas acordadas du 20 novembre 1573 et du 20 novembre 1574, ordonne à tous les inquisiteurs d'appliquer au délit de simple fornication la même procédure que pour l'hérésie. A la suite du concile de Trente, il fallait défendre le sacrement du mariage et réformer les croyances des populations vieilles-chrétiennes en matière de sexualité. Écoutons quelques témoignages.

Une femme vint témoigner contre Mosén Estevan Garaffa, chanoine de Santa Cristina à Jaca car, en juin 1575, lors d'une discussion sur les femmes avec un autre ecclésiastique, il avait déclaré : "juro a Dios que si yo pensase cumplir con las mugeres, que de un año a esta parte abría más de diez, y diziendo el testigo, y el alma ? respondió, cuerpo de Dios que por esso no va ninguno al infierno " (Annexe 3). Miguel de Grey, habitant de Pitilla, affirmait en parlant de choses profanes et obscènes que personne n'allait en Enfer pour avoir entretenu une relation charnelle avec une femme célibataire (Annexe 3, n 56). Le laboureur Joan de Gordún, habitant d'Ejea de los Caballeros, n'éprouvait aucune mauvaise conscience à fréquenter les prostituées de Saragosse. Après tout, ne payait-il pas un service ? :

en 1570, yendo por Çaragoça, dixo el dicho Joan de Gordún al testigo, vamos a las putas, y el testigo le dixo que si a las putas yva y se moría sin hazer descargo dello, que se yva al infierno, y el reo respondió que con las putas del burdel que no era pecado (Annexe 3, $\mathrm{n}^{\circ} 72$ ).

22. Ibidem, p. 318. 
Les délits mineurs représentent $27,7 \%$ de l'ensemble des dénonciations. Ils englobent la contrebande (19\%), la superstition (7\%) et l'opposition au Saint-Office $(1,7 \%)$.

Avec les tribunaux de Catalogne, de Navarre et de Galice, le tribunal de Saragosse était le seul à avoir la juridiction sur le délit de contrebande qui concernait principalement le trafic de chevaux entre l'Aragon et le Béarn. Comme le souligne François Gomez,

La législation sanctionnant ces délits apparut en 1564 lors des Cortès de Monzón. À cette époque, le contrôle de ce trafic se justifiait par la rareté des chevaux qui se trouvaient dans ce royaume. En 1573, on inséra une clause dans l'édit de foi qui invitait les chrétiens à dénoncer tous ceux qui vendaient, passaient ou aidaient à passer des chevaux dans des royaumes protestants (Béarn et Gascogne). Le 25 août 1573, la réglementation se fit beaucoup plus précise puisqu'on obligea les vendeurs de chevaux à tenir un registre extrêmement rigoureux sur le nombre, l'origine et la destination de leurs chevaux. A partir de cette date, quiconque outrepassait ces interdictions était considéré comme hérétique et passible de la peine capitale. La justification de tels délits se fondait sur le fait que les chevaux pouvaient représenter un "matériel de guerre » fourni aux armées hérétiques voisines. Par ce tortueux stratagème, l'Inquisition engloba le trafic des chevaux dans sa juridiction ${ }^{23}$.

Au mois de juin 1575, Pedro de Mur, habitant de Latras, Joan Pérez Zanfolla, habitant d'Escarrilla et Antón Pérez, habitant de Quinto, vendirent deux chevaux pour la somme de quarante écus à un béarnais dans l'auberge de Peyranera (Annexe 3, n 5). Joan Claber, familier du Saint-Office à Torla, arrondissait ses fins de mois en trafiquant avec la France. Le témoin qui dépose contre lui, prend bien soin de préciser qu'il vendait des chevaux à des catholiques français pour l'exempter de tout soupçon d'hérésie :
por la feria de Sant Martín de 1573 passó doze cavallos a Francia en compañia de otros, y los nueve entregó a cuyos eran, y los tres que eran suyos vendió a ciertos capitanes franceses cathólicos, y que rogándole un luterano que le pasasse uno, respondió que no lo haría aunque le diesse toda España porque era luterano (Annexe 3, $\mathrm{n}^{\circ}$ 19).

Parfois la contrebande ne portait pas sur des chevaux mais sur des armes ou de la poudre (salpêtre). Un témoin raconte qu'en 1574, Joan Sánchez,

23. F. Gómez, Le tribunal du Saint-Office de Saragosse..., op. cit., p. 197. 
muletier à Canfranc, avait utilisé l'un de ses domestiques pour faire parvenir des armes à un capitaine protestant du Béarn :

un criado del dicho Joan Sánchez sacaba unas cerrajas de arcabuz a Bearne, y el testigo se las quitó. Y el dicho Joan Sánchez le importunó que se las volviesse que estava obligado a darlas a un capitán luterano aunque le costassen 300 escudos, y diziéndole que estavan vedadas por el Santo Oficio, dixo que no se le dava nada (Annexe 3, n 32).

Le délit de superstition regroupe à la fois les sorcières, les devins, les guérisseurs, les astrologues et les chercheurs de trésors. La plupart du temps, les accusés sont des femmes de condition sociale modeste ayant un faible niveau d'instruction. Comme pour le blasphème, le délit de superstition était un délit mixte qui relevait aussi bien de la justice civile et ecclésiastique que de la justice inquisitoriale. C'est à partir de 1520 que les édits de foi commencent à l'inclure dans la liste des crimes qui relèvent de l'hérésie. L'Inquisition intervient dès lors qu'il y a eu pacte explicite ou implicite avec le diable, atteinte au libre arbitre de la personne et utilisation d'objets sacrés.

María Laguna, que nous avons évoquée plus haut pour sa conduite sacrilège, déclara aux inquisiteurs qu'elle avait été initiée dans l'art de la sorcellerie par une autre femme, qu'elle avait empoisonné des hommes à l'aide de potions magiques et qu'elle avait eu une relation sexuelle contre nature avec Belzébuth lors d'un sabbat. María Jiménez, habitante de La Rosa, confia à un témoin qu'elle s'était réfugiée dans la montagne avec des amies pour y conclure un pacte avec le démon à qui elle donna une goutte de sang de son petit doigt (Annxe 3, $\mathrm{n}^{\circ} 12$ ). Certains habitants de Sallent faisaient appel aux services de María Gasca, surnommée la adivina, en cas de maladies et d'envoûtements. L'un des témoins alla la voir pour résoudre un problème de sexualité conjugale, mais à l'entendre, le remède fut loin d'être efficace :

dize otro testigo que no pudiendo tener acceso carnal a su muger entendiendo que estavan ligados, fueron a la dicha Gasca, que llaman la adevina, para que lo remediasse, la qual dixo que los avian ligado con cuatro palabras a la puerta de la yglesia, y que a ella se le avía olvidado una palabra y que procuraria de acordarse della, y encerró a la dicha muger ligada en su aposento y le dixo que le diesse pelos de las partes bergonçosas delanteras, los quales puso en una escudilla sobre unas brasas, y les andubo meneando con la punta de un cuchillo hasta que se hizieron polvos y los mezcló con vinagre, y la hizo lavar con ello el pecho y la barriga y las partes bergonçosas, pero que no pudieron tener acceso el uno con el otro (Annexe $3, \mathrm{n}^{\circ} 7$ ). 
Le curé de Saragosse, Mosén Pex, se livrait à d'étranges pratiques, en compagnie d'un morisque et de deux autres ecclésiastiques. Il s'amusait à creuser dans le sol pour y déterrer les soi-disant trésors enfouis par les arabes, les goths ou les gens du Moyen Âge (Annexe 3, n 63).

Deux personnes, enfin, furent dénoncées pour entrave à l'action du SaintOffice. L'évêque de Jaca, que nous avons déjà évoqué, avait tenté d'usuper les fonctions inquisitoriales en déclarant qu'il pouvait juger du crime d'hérésie au même titre que n'importe quel juge apostolique et en s'octroyant le titre de grand inquisiteur (inquisidor mayor) (Annexe 3, $\mathrm{n}^{\circ} 3$ ). Malgré les injonctions des jurats d'Aragués del Puerto, le teniente de alcaide Gil de Ypas refusa d'héberger l'inquisiteur en visite dans sa maison, d'assister à la publication de l'édit de foi et de se rendre à la messe (Annexe $3, \mathrm{n}^{\circ} 35$ ).

Les hérésies formelles représentent 17,3\% del'ensemble des dénonciations. En dehors de sept morisques accusés d'observer la fête du Ramadan et de deux judéo-convers dénoncés, l'un pour circoncision (?) et l'autre, pour tiédeur en matière de foi religieuse, les autres accusations concernent le délit de luthéranisme.

L'hérésie protestante se trouve au coeur même de la stratégie déployée par l'Inquisition de Saragosse dès les années 1560. François Gómez défend la thèse selon laquelle le protestantisme en Aragon serait un problème de frontière et de voisinage plutôt qu'un problème religieux. Il a tout à fait raison, mais il ne faut pas oublier aussi qu'il s'agit d'un problème de politique nationale et internationale. C'est l'époque, en effet, où l'Espagne et le Pape se félicitent du massacre de la Saint-Barthélémy survenu le 24 août 1572 et où Philippe II appuie la Ligue catholique dirigée par Henri III et le clan nobiliaire des Guise, soutien qui aboutira en décembre 1584 au traité de Joinville qui prévoyait la formation d'une Sainte Ligue pour lutter contre l'hérésie en France et aux Pays-Bas et une aide financière espagnole de 50000 écus par mois pour l'écrasement en France du parti réformé ${ }^{24}$. Sous la rubrique de luthéranisme, l'Inquisition rangeait des confessions aussi variées que l'évangélisme, le calvinisme ou l'anglicanisme. L'utilisation du luthéranisme à des fins de propagande politique n'avait cure des subtilités théologiques. A travers les autodafés de Séville et de Valladolid (1558-1559) et l'arrestation de l'archevêque de Tolède, Carranza, le Saint-Office entendait brandir le spectre de Luther comme l'hérésiarque par antonomase, favoriser l'émergence d'un sentiment d'identité nationale

24. B. Bennassar et J. Jacquart, Le XVIe siècle, Paris, Armand Colin, 1997, pp. 289-296. 
fondée sur la fidélité à l'Eglise catholique, apostolique et romaine et diffuser une idéologie xénophobe parmi la population. Le protestantisme c'était non seulement Luther mais également l'étranger. Dans le cas aragonais, il s'agissait, en quelque sorte, d'une hérésie d'importation, rendue possible à travers les nombreux contacts établis par les populations de chaque côté de la frontière.

Mais qui sont ces luthériens français dont parlent les inquisiteurs de Saragosse ? Certainement pas des luthériens mais des huguenots d'obédience calviniste. Pour preuve, ce témoignage recueilli contre un dénommé Colet, béarnais du Val de Aspa qui avait participé aux guerres de religion contre les catholiques aux côtés du baron de Ros :

(en 1563) baptizando una criatura el vicario de Urdos conforme al rito de la yglesia cathólica romana y siendo compadre un tío del dicho Colet, no quiso entrar en la yglesia, y desde afuera dixo, qué? a los de la justicia de los uganaos que baptizaban al modo de la yglesia romana?, y se burlaba haziendo gestos dando a entender que aquello no valía nada ni era bueno hazerlo (Annexe 3, $\mathrm{n}^{\circ} 39$ ).

En 1562, au moment où s'ouvrent les premières guerres de religion, on compte en France plus de 670 Eglises pourvues de pasteurs et autant de petites communautés sans pasteur fixe. Les réformés que l'on appelle " ceux de la religion " représentent environ deux millions, c'est-à-dire $11 \%$ de la population globale. Ces communautés réformées sont très fortement concentrées dans l'espace occitan : Guyenne, Gascogne, Béarn, Languedoc, Vivarais, Dauphiné ; espace prolongé à l'ouest par l'Aunis, la Saintonge, le sud du Poitou et les pays de la Loire. On y trouve des clercs et des juristes, des artisans, des marchands, des écrivains et des artistes, mais également des membres de la haute noblesse comme les Bourbon, les Châtillon, les Rohan, etc., ainsi qu'une masse non négligeable de gentilshommes ${ }^{25}$.

Dans le Béarn, la Réforme est liée à la figure d'Henri IV et de sa mère Jeanne d'Albret. Après la défaite de Jarnac en 1569, les troupes royales de Catherine de Médicis entreprirent une offensive de grande envergure contre les huguenots du Béarn. Jeanne d'Albret eut alors recours au chef de sa garde écossaise, Montgoméry, pour mener à bien la contre-offensive. Il ne fallut que trois semaines à cette armée de reconquête pour mettre en déroute les troupes de Catherine de Médicis qui durent aussitôt lever le siège de

25. H. Dubief et J. Poujol, La France protestante. Histoire et lieux de mémoire, Max Chaleil Editeur, 1992, pp. 19-53. 
Navarrenx. À l'issue de cette libération, le protestantisme devint la religion dominante en Béarn pendant un demi-siècle et Pau, Sauveterre, Nay et Oloron se transformèrent en véritables bastions réformés.

D'un point de vue doctrinal, le calvinisme obéit, tout en s'en éloignant sur certains points, aux grands principes de la Réforme luthérienne. Il se caractérise par :

- l'éradication des pratiques idolâtres et magiques qui ont défiguré la vérité chrétienne ;

- le refus d'aller à l'Eglise pour assister à la messe ou se confesser vocalement. L'Église réformée est composée de pasteurs ou ministres, serviteurs de la parole de Dieu, désignés par la communauté, et d'un collège d'anciens, laïcs chargés de veiller sur la discipline des fidèles. Le culte protestant, célébré le dimanche dans le temple, s'articule essentiellement autour de la lecture de la Bible, du prêche, de la prière et du chant des psaumes. Quatre cènes annuelles sont prévues ;

- la désacralisation du temps, des lieux, des objets, des vivants et des morts, et des sacrements eux-mêmes (mariage, extrême-onction, eucharistie, pénitence, ordre, confirmation). Deux sacrements seulement subsistent : la cène et le baptême ;

- l'abolition de tous les intermédiaires, de tous les médiateurs entre Dieu et les hommes, à l'exception de Jésus-Christ ;

- la négation de l'existence du purgatoire ;

- la justification par la foi et non par les œuvres et les mérites. L'homme, pécheur par nature, est justifié par la foi que Dieu lui accorde gratuitement ;

- la suppression de la séparation entre clercs et laïcs (théorie du sacerdoce universel) ;

- la négation de l'autorité papale, de la hiérarchie ecclésiastique, avec son système de bulles et d'indulgences ;

- le non-respect des jours de jeûne (Carême y compris).

À cet égard, la confession de Ramonet de Hostau, meunier originaire de la Seuba en Béarn, constitue un véritable acte d'adhésion à la fois réformée :

...vino de su tierra a Jaca a confesar y confesó que en compañía de otros hizo la protestaçión que los luteranos acostumbran hazer en manos del ministre (sic) dellos, prometiendo de renunciar la missa y los clérigos y obispos y la ydolatría de los cathólicos, y que los santos ni nuestra señora no tenían pujança, y después hiço la cena con los dichos luteranos tres o quatro vezes, y oyó los prexes (sic) dellos, donde dezian que la missa era abuso y que la ostia era un pedaço de pan y que no aprobechaba aquello cosa alguna, y que no avía purgatorio, que en este mundo era 


\begin{abstract}
el purgatorio quando tenian dolençias y trabajos, y que no avía que rogar por los muertos, y que si creyan en la missa que eran ypócritas, y que lo de la confesión no era sino abuso y dar cuenta a los clérigos de sus bellaquerías, que no podian perdonar nada. Y que los sanctos no valian cosa, ni se les avia de rogar porque no tenian pujança alguna, ni tampoco la Virgen María. Y que el papa no era sino como otro hombre que no podia perdonar nada; y que las bullas y perdones que da, no aprobechaban ni valian nada, que no era sino un Antechristo. $Y$ que se podia comer carne todos los días sin dificultad, y que no se avian de guardar otras fiestas sino sólo el domingo. $Y$ que tubo y creyó dichos errores pensando que eran buenos y se salvaba en creherlos. Y que comió carne como los luteranos la comian en dias probibidos creyendo que en todos días se podía comer sin escrúpulos (Annexe 3, n 14).
\end{abstract}

Les délits sexuels englobent la bestialité, la sodomie et la bigamie et représentent 9,8\% de l'ensemble. Il s'agit-là, avec le délit de contrebande de chevaux, d'une spécialité aragonaise, les délits contre nature étant dans la couronne de Castille du ressort exclusif des justices ecclésiastique et civile.

Une pragmatique des Rois Catholiques du 14 janvier 1505 fit entrer les délits de sodomie et de bestialité dans la sphère inquisitoriale, mais il fallut attendre le bref papal du 24 février 1524 adressé aux inquisiteurs aragonais, catalans et valenciens pour que le Saint-Office de la couronne d'Aragon pût se saisir du pecado nefando. La sodomie et la bestialité étaient considérées comme des délits contre nature car ils allaient à l'encontre de l'ordre naturel et divin ${ }^{26}$. Dans la catégorie des péchés de chair, définie par saint Grégoire, elles incarnaient les deux formes les plus abominables du péché de luxure ${ }^{27}$. Les inquisiteurs faisaient la distinction entre trois formes de sodomie : la sodomie " parfaite » entre deux personnes du même sexe, la sodomie « imparfaite " entre personnes du sexe opposé et la sodomie bestiale perpétrée par des hommes sur des mules, des ânesses, des poules, etc. Ils se souciaient également de savoir s'il y avait eu ou non émission de semence humaine (polución). La bigamie, quant à elle, relevait autant de la justice civile et ecclésiastique que de la justice inquisitoriale. Elle allait à l'encontre du sacrement du mariage chrétien fondé sur la monogamie, l'indissolubilité de l'union et la fidélité des époux. Le concile de Trente, dans sa session XXIV du 11 novembre 1563, définissait le bigame en ces termes :

26. Sur ces affaires de "pecado nefando", on peut consulter Raphaël Carrasco : Inquisición y represión sexual en Valencia. Historia de los sodomitas (1565-1785), Barcelone, Laertes, 1985, et «Las torpezas nefandas. El castigo de la sodomía», Debats, 2-3, 1982, pp. 32-39.

27. B. Bennassar, L'Inquisition espagnole, op. cit., pp. 330 et ss. 
Nombreux sont qui vont d'un lieu à un autre et n'ont pas de domicile fixe. En hommes pervers qu'ils sont, après avoir abandonné leur première épouse, du vivant de celle-ci, ils se marient à une autre et souvent même à plusieurs en divers lieux. Le saint concile, désirant porter remède à ce mal, avertit paternellement tous ceux que cela concerne de ne pas admettre facilement au mariage ce genre d'hommes vagabonds. Il exhorte aussi les magistrats séculiers à les réprimer sévèrement. Il enjoint aux curés de n'assister au mariage de ces hommes qu'après avoir fait au préalable une enquête sérieuse et, la chose ayant été déférée à l'Ordinaire, après avoir obtenu de celuici la permission d'agir ainsi ${ }^{28}$.

Mosén Nicolás Clarchet accuse l'archidiacre de Gorga, don Hierónimo de Urries, alias Gabarre, d'avoir eu des relations sexuelles contre nature avec lui, alors qu'il était étudiant à l'université de Huesca. La scène qu'il décrit a lieu en 1567 au domicile de l'archidiacre. Aucun détail n’y est épargné :

Fue una noche a casa del dicho arcediano e hizo colaçión con él, y queriéndose yr no le dexó diziéndole que era noche y que con él dormiria, $y$ ansi se acostaron juntos en una cama y dicho arcediano luego abrazó y besó al testigo y se apretó con él cara a cara y andaba meneando su miembro genital con la mano y después el del testigo, y ajuntados el uno con el otro cara a cara puestos de lado, el dicho arcediano vino en poluçión. Hecho esto parlaron un rato, y después tornó dicho arcediano a tomarse su miembro y meneallo y se juntó con el testigo diziéndole que se volviesse de espaldas y lo fue a abrazar, y como el testigo no le respondia le preguntó si dormía, y diziéndole que no, lo abrazó y besó y andubo meneando su miembro y el cuerpo y empuxando para delante, $y$ le daba topetones por las piernas con el miembro, $y$ que no hizo fuerça para volverlo ni sabe si viniesse esta vez en polución (Annexe $3, \mathrm{n}^{\circ} 1$ ).

Joan de Assún, habitant de Nabassa fut surpris dans un champ par une femme qui l'avait vu «tener acceso carnal con una asna, teniéndola asida por detrás y muy pegado a ella» (Annexe 3, $\mathrm{n}^{\circ} 2$ ). Trois hommes furent dénoncés pour délit de bigamie. On les soupçonnait d'avoir une épouse en Aragon et une autre en Béarn.

En guise de conclusion, nous dirons que cette enquête menée à partir d'une visite de district du tribunal de Saragosse, confirme ce que l'on savait déjà sur l'Inquisition à l'époque de Philippe II, à savoir son identification étroite à l'État contre-réformiste et providentiel des Habsbourg.

28. G. Alberigo (dir.), Les conciles oecuméniques, t. 2, Paris, CERF, 1994, p. 758. 
Entre le 17 août et le 13 décembre 1575, l'inquisiteur Diego de Haedo parcourut plus de $400 \mathrm{kms}$ et visita plus de quinze localités dans la partie nord-est de son district et dans le Haut-Aragon limitrophe avec le Béarn français, véritable bastion huguenot. Pendant la visite, 105 personnes furent dénoncées par la population et on instruisit 29 procès contre des blasphémateurs issus en majorité du monde rural et du clergé.

Le tribunal de Saragosse abandonna momentanément la lutte contre l'hérésie mahométane, son principal cheval de bataille, pour se pencher sur les délits de paroles (45,2\%), les délits mineurs (27,7\%), le luthéranisme $(9,6 \%)$ et les délits sexuels $(9,8 \%)$. Si l'on ajoute au luthéranisme, la plupart des propositions et la contrebande de chevaux et d'armes avec le Béarn, on s'aperçoit que l'hérésie protestante arrive largement en tête. Celleci représente non seulement un problème de frontière mais également un problème religieux et politique.

À la suite du concile de Trente, l'Inquisition aragonaise tenta de mettre en place un modèle religieux et sexuel basé sur la stricte adhésion au catholicisme romain et la défense du mariage chrétien. Cela dit, a-t-elle réussi à faire taire les langues trop bien pendues des vieux-chrétiens et à freiner les débordements lubriques des ecclésiastiques sodomites et des culs-terreux zoophiles ? Rien n'est moins sûr, mais on ne peut nier qu'elle a largement contribué à la diffusion de la Contre-Réforme. 
DIEGO DE HAEDO DANS LE DISTRICT INQUISITORIAL DE SARAGOSSE (I 575)

\section{ANNEXES}

\section{1) Chronologie et itinéraire de la visite du licencié Diego de Haedo :}

- 17/08/1575 : départ de l'inquisiteur pour Jaca.

- 19/08/1575: arrivée à Jaca où il séjourne quinze jours (distance Saragossse-Jaca : environ $140 \mathrm{kms})$.

- 3/09/1575 : arrivée à Biescas où il reste quatre jours (distance JacaBiescas : environ $30 \mathrm{kms})$.

$-7 / 09 / 1575$ : arrivée à Torla, près de la frontière française, où il reste dix jours (distance Biescas-Torla : environ $20 \mathrm{kms}$ ).

- 17/09/1575 : arrivée à Sallent où il reste sept jours (distance TorlaSallent : environ $32 \mathrm{kms})$.

$-24 / 09 / 1575$ : arrivée à Canfranc où il reste sept jours (distance SallentCanfranc : environ $16 \mathrm{kms})$.

$-1 / 10 / 1575$ : arrivée à Sinués où il reste deux jours (distance CanfrancSinués : environ $16 \mathrm{kms})$.

$-3 / 10 / 1575$ : arrivée à Aragués del Puerto où il reste cinq jours (distance Sinués-Aragués : environ $8 \mathrm{kms}$ ).

- 8/10/1575 : arrivée à Hecho où il reste sept jours (distance AraguésHecho : environ $8 \mathrm{kms}$ ).

- 15/10/1575 : arrivée à Ansó où il reste sept jours (distance HechoAnsó : environ $8 \mathrm{kms}$ ).

- 22/10/1575 : arrivée à Berdún où il reste huit jours (distance AnsóBerdún : environ $20 \mathrm{kms})$.

- 25/10/1575 : arrivée à Biel où il reste deux jours (distance BerdúnBiel : environ $36 \mathrm{kms})$.

- 27/10/1575 : arrivée à Luesia où il reste trois jours (distance BielLuesia : environ $12 \mathrm{kms})$.

- 30/10/1575 : l'inquisiteur retourne à Jaca où il reste vingt-sept jours (distance Luesia-Jaca : environ $50 \mathrm{kms}$ ).

- 26/11/1575 : arrivée à Sos, à la frontière navarraise, où il reste trois jours (distance Jaca-Sos : environ $72 \mathrm{kms}$ ).

$-29 / 11 / 1575$ : arrivée à Uncastillo où il reste quatre jours (distance SosUncastillo : environ $20 \mathrm{kms}$ ).

$-3 / 12 / 1575$ : arrivée à Sábada où il reste quatre jours (distance UncastilloSádaba : environ $14 \mathrm{kms})$.

$-7 / 12 / 1575$ : arrivée à Exea de los Caballeros où il reste six jours (distance Sádaba-Exea : environ $20 \mathrm{kms})$. 

$\mathrm{kms})$.

- 13/12/1575: arrivée à Tauste (distance Exea-Tauste: environ 24

Durée de la visite : 4 mois dont un mois et demi dans la ville de Jaca. Distance parcourue : $400 \mathrm{kms}$.

Pendant le premier séjour de l'inquisiteur à Jaca, un commissaire du Saint-Office est chargé d'inspecter Ayerbe et ses alentours : Murillo, Sancta Engracia, Aguero, Loharre, Biscarrués, Puentedeluna, Ardissa, Sasa de Marguello, Sancta Olalia, Los Corrales, Aniés, Bentue, Rafal et Riglos.

\section{2) Dates et lieux de publication de l'Edit de la Foi.}

- 21/08/1575: (Jaca) Asiso, Ypas, Atarés, Guasillo, Bergosa, Grazamupuel, Larrés, Sancta Cruz, Guasa, Bandrés, Camias, Novés, Barraguás, Martellue, La Rosa, Asín, Villanobilla, Araguás del Solano, Badaguas, Varos, Yosa de Garcipollera, Besros, Xarlata, Franco, Nabasilla, Nabassa, Sasal, Ascares, Bernués, Orante, Espuéndolas, Pardinilla, Acumuez, Borrés, Cartirana, Ulle.

- 4/09/1575 : (Biescas) Senegue, Sorripas, Berbusa, Aniello, Escuez, Casuas, Sosín, Larrede, Oliván, Yesero, Latras, Xabierre, Satué, Aso, Yosa de Sobremonte, Betes, Afur, Gabín, Oros alto, Oros baxo, Barbenuta, Ezpierre.

- 8/09/1575 : (Torla) Broto, Faxen, Asín, Bergua, Cortillas, Sasa, Cillas, Ayerbe, Aysuela, Yosa, Buesa, Utal, Escartín, Lejarán, Sarlisse, Linas.

- 18/09/1575: (Sallent) Panticosa, San Dimes, Escarrilla, Lanuça, Tramacastilla, El Pueyo, Foz, Piedrahita, Bubas.

-2/10/1575: (Sinués) Borao, Asposa, Aysa.

- 9/10/1575 : (Echo) Siresa, Urdués.

- 16/10/1575: (Anso) Frago.

- 22/10/1575: (Berdún) Viniés, Maxones, Bagués, Vaylo (Bailo), Xabierregay, Sancta Cilia, Alastuey, Guertolo, Larrués, Mianos, Vinagua, Sancta Engracia, Arbués, Ayso, Villareal, Artieda, Larbes, Embún, Martes. $-26 / 10 / 1575$ : (Biel).

- 28/10/1575 : (Luesia) Longas, Fuencalderas, Sanct Felizes, Asín, El Frago, Salinas, Salvatierra.

- 27/11/1575 : (Sos) Nabardún, Urriés, Pitilla, Isuerre, Ruesca, Lobera, Undués de Cabolerda, Pintano, Undués Pintano.

- 29/11/1575: (Uncastillo) Malpica.

- 4/12/1575 : (Sádaba) Layana, Viota, Castiliscar. 
DIEGO DE HAEDO DANS LE DISTRICT INQUISITORIAL DE SARAGOSSE (I 575)

- 8/12/1575 : (Exea de los Caballeros) Luna, Erla, Valpalmas, Ribas, Farasdués.

- 14/12/1575 : (Tauste) Pradilla, Remolinos.

3) Liste des personnes dénoncées et condamnées par l'Inquisition au cours de la visite de 1575 .

Légende : MAH (mahométisme) ; JUD (judaïsme) ; LUTH (luthéranisme) ; BLASPH (blasphème) ; PROP (propositions hérétiques); SEX (propositions à caractère sexuel); SOD (sodomie) ; BEST (bestialité); BIG (bigamie) ; CONT (contrebande) ; SO (opposition au Saint-Office); SUP (superstition).

1) Don Hierónimo de Urries, alias Gabarre, archidiacre de Gorga (SOD).

2) Joan de Assún, habitant de Nabassa (BEST).

3) Don Pedro del Frago, évêque de Jaca (PROP -eucharistie, confession- ; $\mathrm{SO})$.

4) Andreba Garcés, habitante de Jaca (SUP).

5) Pedro de Mur, habitant de Latras ; Joan Pérez Zanfolla, habitant d'Escarrilla et Antón Pérez, habitant de Quinto (CONT).

6) Pedro de Barriomayor, habitant de Yosa (PROP -immortalité de l'âme-).

7) María Gasca, habitante de Sallent (SUP).

8) María Laguna, habitante d'Azín (PROP -sacrilège- ; SUP).

9) Joan de Ayneto, habitant d'Ayneto (BLASPH ; CONT ; PROP sacrilège, culte des saints et des images).

10) Pedro de Vergua, habitant de Sardas (CONT).

11) Miguel Beseos, habitant de Beseos (PROP -immortalité de l'âme-).

12) María Jiménez, habitante de La Rosa (SUP).

13) Orosia Escartín, habitante de Varos (BLASPH).

14) Ramonet de Hostau, meunier, habitant de la Seuba (LUTH).

15) Joan de Arras, tailleur et marchand, habitant de Gan (LUTH).

16) Pedro Marín, habitant de Cartirana (BEST).

17) Martín Martón, habitant de Sallent (CONT).

18) Martín de Bergua, seigneur d'Espín (CONT ; LUTH).

19) Joan Claber, familier du Saint-Office à Torla (CONT).

20) Ramón Negre, français (PROP -indulgences, bulles-).

21) Joan de Marco, habitant de Fraxen (PROP -messes pour les âmes du purgatoire-). 
22) Guillem de Sancta Coloma, charpentier béarnais, habitant de Linas (LUTH ; PROP -purgatoire, messe, oeuvres, baptême-).

23) Ramón Joan et Arnao Cortada, habitants de Luz (France) (BLASPH ; PROP -vierge Marie-).

24) Vicente de Bergua, habitant de Bergua (CONT ; LUTH).

25) Catalina de Bergua, habitante de Bergua (BLASPH).

26) Bernard Campra, habitant de Solon en Gascogne (BLASPH).

27) Pedro Borbón, habitant d'Aynsa (BLASPH).

28) Matheo Bescos, laboureur, habitant de La Almunia de Montaragón (SEX).

29) Francisco Artillón, morisque, habitant de Torres de Barbués (MAH).

30) Guallart de Casabiella, habitant de Biela (Béarn) (CONT).

31) Gerónimo Diza, Francisco Garrote, Juan Diza, Miguel Diza et Alexandre Diza, morisques (MAH).

32) Joan Sánchez, muletier, habitant de Canfranc (CONT ; PROP -bulles de la Croisade- ; LUTH).

33) Joan de Esporrín, marchand, Joan de Oluc et un jeune béarnais, habitants d'Oloron (LUTH).

34) Joan de Santa Engracia, habitant d'Aragués (BIG).

35) Gil de Ypas, habitant d'Aragués (BLASPH ; SO).

36) Pedro Peyran, habitant de Hecho (CONT).

37) Joan del Pueyo menor, habitant de Hecho (CONT).

38) Joan Peyran, habitant de Hecho (CONT).

39) Colet, béarnais du Val de Aspa (LUTH).

40) Domingo Ximénez, habitant de Ciresa (PROP -sacrilège, culte des saints-).

41) Mosén Domingo Puyo, mayor, habitant d'Anso (SUP).

42) Monic, béarnais, Joan de Atares, Francisco de Atares, habitants de Larves, Fabián Ximénez, habitant de Ciresa, Domingo Ximénez, habitant de Bailo, Martín de Assín, habitant de Longas (CONT).

43) Joan López et Joan Ximénez, habitants de Bailo (BLASPH).

44) Pedro Alastuey, habitant de Pintano (BLASPH).

45) Joan de Supravilla, a. Verdol, habitant de Biel (PROP -âmes du purgatoire- ; BLASPH).

46) Joan Francés, habitant de Luesia (LUTH).

47) Bartolomé de Luna, habitant d'Alcalá de Gurrea (BEST).

48) Joan de Camias, laboureur, habitant de Santa Cilia (PROP -virginité de Marie-).

49) Andrés, hombre pobre, habitant de Jaca (BLASPH ; PROP -images, saints, confession-). 
50) Blasco de Asín, seigneur d'Aruex (CONT).

51) Miguel de Bernués, habitant de Vinaqua (PROP -défunts-).

52) Mosén Pedro Guallart, grand chapelain de la seo de Jaca (PROP -messes, dévotions-).

53) Francés Ruiz, alcaide de Xabierrelatre (CONT).

54) Pedro de Bergua, habitant de Yebra (CONT).

55) Joan de Sento, laboureur gascon, habitant de Uncastillo (PROP -enfer-).

56) Miguel de Grey, habitant de Pitilla (SEX).

57) Un savetier de Saragosse (SEX).

58) Pedro de Naquesa, habitant d'Undués Pintano (SEX).

59) Une gasconne d'Uncastillo (PROP -oraisons-).

60) Joana épouse de Fortanet, habitante d'Uncastillo (SUP).

61) Un fils de Martín de Longas, habitant d'Uncastillo (JUD).

62) Mosén Miguel Felizes, habitant d'Uncastillo (JUD).

63) Mosén Pex, curé de Saragosse (SUP).

64) Joannes Viscayno, habitant de Monzón (SEX).

65) Le vicaire de Castejón (PROP -résurrection-).

66) Pedro Botia, habitant de Sádaba (PROP -âme, purgatoire-).

67) Joan Rodríguez, maître d'orgues (PROP).

68) Arnao, berger gascon (PROP -bulles-).

69) María Sinués, habitante d'Ejea de los Caballeros (PROP -messe, enfer-).

70) Ramón, journalier gascon, habitant d'Ejea (PROP -sacrilège-).

71) Seubat, apprenti laboureur, habitant d'Ejea (PROP -saints, hostie-).

72) Joan de Gordún, laboureur, habitant d'Ejea (SEX).

73) Pedro Gascón, berger, habitant d'Ejea (PROP -eucharistie-).

74) Pedro Linaje, habitant de Pitilla (CONT).

75) Joan López del Vaylo, habitant d'Ejea (PROP -sacrilège-).

76) Gabriel de Arcadí, morisque, habitant de Lurzemic (MAH).

77) Joan Gómez, juglar, habitant de Tauste (PROP -bulles-).

78) Agustín de Santangel, habitant de Saragosse et Cristóbal Garcés, habitant de Tauste (BLASPH).

79) Joan Sant Clemente, a. el fraylet de Fontellas (BEST).

80) Francisco Segura, habitant de Montemediano (BIG).

81) Francisco Sescosa et Salvador Torrero, habitants d'Ayerve (SOD).

82) Antón de Vera, tailleur français (LUTH).

83) Guillén de la Guarda, français, habitant d'Ayerve (LUTH).

84) Estevan Apilluelo, habitant de Santa Olalia (SEX ; BLASPH). 
85) Beltrán, mozo francés, habitant d'Ayerve (BEST).

86) Catalina Labedan, habitante de Rafal (SUP).

87) Bernard Jaymot, barbier gascon, habitant de Rafal (BIG).

88) Miguel Torrero, tailleur, habitant d'Ayerve (PROP-virginité de Marie-).

\section{4) Liste des principaux blasphèmes et des primes infligées aux blasphémateurs.}

1) Domingo Segura, vecino de Orna. Decir descreo de Dios, reniego de Dios y pese a Dios. Causa suspendida.

2) Gerónimo Xabierre, vecino de Jaca. Decir descreo de Dios. Causa suspendida.

3) Miguel de Gabín, alcaide de Oliván. Decir reniego de Dios y de todos los santos que hay en el paraíso, tomo al diablo por señor. Causa suspendida.

4) Pedro Escartín mayor, labrador. Decir descreo de Dios, "pese a Dios con el bellaco». Causa suspendida.

5) Joan Desporrín mayor. Decir reniego de Dios. Causa suspendida.

6) Pedro López, labrador, vecino de Acumuer. Decir reniego de Dios o descreo de Dios. Causa suspendida.

7) Joan Pelegrín, labrador, vecino de Yosa. Decir descreo o reniego de Dios. Causa suspendida.

8) Joan de Campana, piquero gascón, vecino de Uncastillo. Por decir descreo de Dios. Causa suspendida.

9) Mosén Domingo Mancho, clérigo, vecino de Anso. Decir descreo de Dios. Causa suspendida.

10) Miguel Mingarro, a. Mingarronet, labrador, vecino de Sallent. Decir descreo y reniego de Dios, voto a Jesucristo que te haré saltar los cascos de la cabeza, por las plagas de Dios, cabeza y cuerpo de Dios. Abjuración de levi, penitencias espirituales, multa de 20 escudos.

11) Sebastián de Latas, pastor, vecino de Ciellas. Decir pesar de Dios, reniego de Dios o de Jesús Nazareno, descreo de Dios, reniego de Dios y de Santa María, cabeza de Dios. Abjuración de levi, penitencias espirituales.

12) Mosén Joan de Bergua, rector de la parroquia de Ianuas (?). Decir reniego y descreo de Dios. Causa suspendida.

13) Pedro de la Torre, ganadero, vecino de Villanueva. Decir pese a Dios, descreo de Dios. Penitencias espirituales, multa de 10 escudos (Hecho, 12/10/1575).

14) Pedro Sagal, labrador, vecino de Santa Cruz de Caseros. Decir descreo de Dios. Penitencias espirituales, multa de 12 escudos (Jaca, 14/11/1575). 
15) Pedro de Sasa, labrador, vecino de Bergua. Decir que «descreía de Dios y de su parte de parayso» y «tomaba al diablo por señor». Penitencias espirituales, multa de 8 escudos (Jaca, 17/11/1575).

16) Miguel de Allue, vecino de Sasa. Decir descreo de Dios. Penitencias espirituales, multa de 12 escudos (Jaca, 14/11/1575).

17) Joan de la Tapia, labrador, vecino de Yxuela. Decir descreo de Dios. Penitencias espirituales, multa de 8 escudos (Jaca, 14/11/1575).

18) Antón Salvador, vecino de Sallent. Decir reniego de Dios y de Santa María, descreo de Dios y pese a Dios. Penitencias espirituales, multa de 10 escudos (Jaca, 17/11/1575).

19) Mosén Pedro Satuc, vicario, vecino de Satuc. Decir reniego de Dios. Penitencias espirituales, multa de 4 ducados (Jaca, 17/11/1575).

20) Joan Martón mayor, vecino de Sallent. Jurar a Dios y a nuestra señora, tomar al diablo por señor, decir "pongome debaxo el culo de Judas si os hago llebar maniatado a Guesca». Abjuración de levi, destierro de dos meses de Sallent, penas espirituales, 50 escudos para gastos del Santo Oficio (Jaca, 17/11/1575).

21) Blasco Gil, labrador, vecino de Aragués. Decir reniego de Dios. Penitencias espirituales (Jaca, 13/11/1575).

22) Martín López, labrador, vecino de Bergua. Decir pese a Dios, descreo de Dios. Penitencias espirituales, multa de 10 escudos (Jaca, 13/11/1575).

23) Joan de Alluc mayor, vecino de Sasa. Penitencias espirituales, multa de 10 escudos (Jaca, 13/11/1575).

24) García Borres, labrador. Decir cuerpo de Dios, plagas y cabeza de Dios (Jaca, 13/11/1575).

25) Antón de Hito, vecino de Aragués. Por decir «dexo a Dios y tomo al diablo por señor». Penitencias espirituales (Jaca, 20/11/1575).

26) Pedro Garafa, labrador, vecino de Ordobes. Decir descreo de Dios. Penitencias espirituales (Jaca, 20/11/1575).

27) Francisco la Costa, tejedor, vecino de Biescas. Decir renuncio a Dios y tomo al diablo por señor. Abjuración de levi, penitencias espirituales, multa de 20 ducados.

28) Joan Palacín, labrador, vecino de Nabasilla. Por blasfemia. Abjuración de levi, destierro por 6 meses, penas espirituales.

29) Salvador de Sinués, ganadero, vecino de Exea de los Caballeros. Jurar a Dios y a los santos. Penitencias espirituales, multa de 24 escudos (Jaca, 20/11/1575). 
